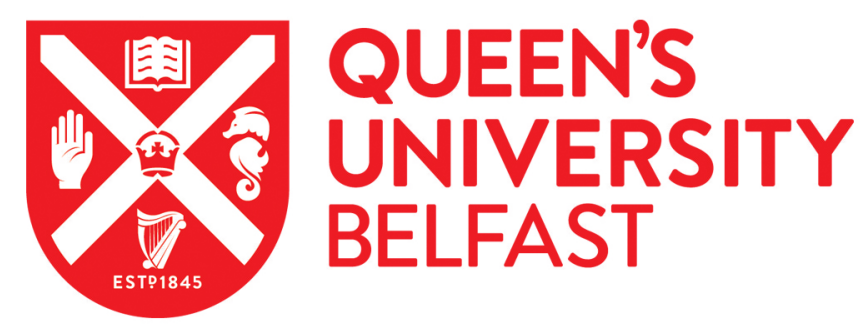

\title{
Plastic cannulae versus metal needle cannulation in haemodialysis: Results of an international survey from the nurse perspective
}

\author{
de Barbieri, I., Sisti, D., Strini, V., Noble, H., Parisotto, M. T., Amatori, S., \& Rocchi, M. B. (2021). Plastic \\ cannulae versus metal needle cannulation in haemodialysis: Results of an international survey from the nurse \\ perspective. The journal of vascular access. https://doi.org/10.1177/11297298211066763
}

Published in:

The journal of vascular access

Document Version:

Peer reviewed version

Queen's University Belfast - Research Portal:

Link to publication record in Queen's University Belfast Research Portal

Publisher rights

(C) 2021 The Authors.

This work is made available online in accordance with the publisher's policies. Please refer to any applicable terms of use of the publisher.

\section{General rights}

Copyright for the publications made accessible via the Queen's University Belfast Research Portal is retained by the author(s) and / or other copyright owners and it is a condition of accessing these publications that users recognise and abide by the legal requirements associated with these rights.

Take down policy

The Research Portal is Queen's institutional repository that provides access to Queen's research output. Every effort has been made to ensure that content in the Research Portal does not infringe any person's rights, or applicable UK laws. If you discover content in the Research Portal that you believe breaches copyright or violates any law, please contact openaccess@qub.ac.uk. 


\title{
Plastic cannulae versus metal needle cannulation in haemodialysis: Results of an international survey from the nurse perspective
}

The Journal of Vascular Access

I-7

(C) The Author(s) 2021 Article reuse guidelines: sagepub.com/journals-permissions DOI: 10.1 | $77 / \mid$ | 2972982 I 1066763 journals.sagepub.com/home/jva (S)AGE

\author{
Ilaria de Barbieri' ${ }^{(D)}$, Davide Sisti², Veronica Strini ${ }^{3}$, Helen Noble $^{4}$, \\ Maria Teresa Parisotto ${ }^{5}$, Stefano Amatori ${ }^{2}$ (D) \\ and Marco BL Rocchi ${ }^{2}$
}

\begin{abstract}
Background: In haemodialysis is key to successfully obtaining cannulation of the arteriovenous fistula (AVF). The literature agrees that cannulation, failing in the initial maturation period, can lead to delayed dialysis, haematoma, scarring, needle phobia and loss of confidence in the cannulator. The introduction of plastic cannulae for haemodialysis has changed cannulation practice positively, preventing frequent complications such as infiltration or trauma. Despite that, most countries have continued to use metal cannulation, in particular in Europe. This study investigates the common use of plastic cannulae versus metal needles for cannulation in dialysis units and explores the implications of focusing on the side effects of cannulation.

Methods: The study is a cross-sectional survey. A questionnaire was created by a team of experts from the European Dialysis Transplant Nurse Association/European Renal Care Association (EDTNA/ERCA) to address the study's aims and sent online to nurse members.

Results: Data collected suggested a strong resistance towards using plastic cannulae, with few respondents claiming to use these cannulas. Most of the respondents were female (74\%), Europeans, working in nephrology for more than 10 years and most worked in the public sector. There was a strong correlation between the use of plastic cannulae and fewer adverse events in elbow located AVF and newly created or fragile AVF.

Conclusions: The results are in line with the current literature. Possible resistance to the use of the plastic device includes the difference in cost between the two devices in favour of metal needles. However, it should be considered that the lower number of adverse events, in particular infiltration and haematoma caused by the metal needle, involves a considerable saving both in money and in terms of time and distress for the patient.
\end{abstract}

\section{Keywords}

Cannulation, haemodialysis, metal needle, nursing, plastic cannulae

Date received: 17 September 2021; accepted: 27 November 2021

\section{Introduction}

In haemodialysis, it is key to successfully obtaining cannulation of the arteriovenous fistula (AVF). There are three modes: via rope ladder (rotation of cannulation site, using the entire length of the cannulation segment), buttonhole (constant site, repeating cannulation at the same site, using the same insertion and same depth) or area technique (puncturing nearby previous cannulation site). For arteriovenous graft (AVG), the preferred cannulation technique is the rope ladder. Scientific evidence supporting the use of buttonholes for AVG has not yet been generated. ${ }^{1}$ It is
'Department of Health Professions, University Hospital of Padova, Padova, Italy

${ }^{2}$ Department of Biomolecular Sciences, Service of Biostatistics, University of Urbino, Urbino, Italy

${ }^{3}$ Projects and Clinical Research Unit, University Hospital of Padova, Padova, Italy

${ }^{4}$ School of Nursing and Midwifery, Queen's University Belfast, Belfast, UK

${ }^{5}$ European Specialist Nurses Organisation, Bruxelles, Belgium

\section{Corresponding author:}

Stefano Amatori, Department of Biomolecular Sciences, Service of Biostatistics, University of Urbino, Piazza Rinascimento 7, Urbino 61029, Italy.

Email: stefano.amatoril@uniurb.it 
essential that cannulation can decrease adverse events, such as the extravasation of the vessel (during cannulation or the haemodialysis session), haematoma, long term aneurysm formation or narrowing of the vessel due to intimal hyperplasia from scars. ${ }^{2}$

Area puncture cannulation techniques and rope ladder ${ }^{3}$ have existed since the 1960s. ${ }^{4}$ The only significant change came in 1973 when cannulation techniques came in the form of a buttonhole or constant site. ${ }^{5}$ The main advantages reported for the buttonhole technique are increased ease of cannulation, fewer failures in puncture, less pain, fewer aneurysms and haematomas. ${ }^{6}$ Since then, there have been many discussions comparing the three techniques. ${ }^{4}$ The literature agrees that cannulation, failing in the initial period of maturation, can lead to delayed dialysis, haematoma, scarring, needle phobia, loss of confidence in the cannulator. Moreover, the costs could increase for a patient home or performing diagnostic imaging to identify the problem or insertion of a central venous catheter (CVC) ${ }^{1,2}$ In addition to the innovations related to cannulation practice, some improvements were linked to the needle length and point-of-care ultrasound for evaluation and guidance cannulation. $^{2}$

Despite that, over the past 50 years, most countries have continued to use metal cannulation. In particular, in Europe, traditional metal needles are the most frequently used device to cannulate an AVF/AVG. ${ }^{7,8}$ However, the increase in diabetes and peripheral vascular disease patients has resulted in an increase in the number of underdeveloped arteriovenous fistulas (AVF) due to arterial calcification, small size of the vessel and poorer therapeutic properties. ${ }^{9}$ These events can significantly affect the nurse's ability to cannulate AVF, particularly when patients are new to haemodialysis due to their frailer vessels.

The introduction of plastic cannulae could decrease many of the problems with cannulation in frail vessels. The use of plastic cannulas started in Japan ${ }^{7,8}$ where plastic haemodialysis cannulas have been available for many years. The plastic cannula has been used in Japan for over 20 years, with about 300,000 patients dialysing three times a week with very little reported evidence of adverse outcomes. ${ }^{10}$ The use of plastic cannulas for AVF was recently introduced in Australia, and initial results reported a reduced risk of infiltration, reduced trauma to tissue and vein and the ability to cannulate difficult and tortuous sections of the AVF. Although there was no evidence at the beginning in the literature to support their use to these types of veins' problem, these data were reasonable to offer patients a better alternative to metal needles. ${ }^{9}$ In Australia, the Center for the Surveillance of Healthcare Related Infections and Prevention and Tuberculosis Control stated that metal needles should not be used for peripheral vascular access in the general hospital population. ${ }^{11}$
Important improvements in patient quality of life and compliance have been noted with the use of plastic cannulae. Du Toit ${ }^{8}$ reported that patients could be cannulated in the antecubital fossa, tortuous parts of the vessels and that patients have been able to move their arms during dialysis without fear of extravasation. There is also a reduced risk of the plastic cannulae entering the vessel's side, moving laterally or during removal. The success of plastic cannulae, particularly associated with the guide of ultrasound, has also been reported ${ }^{7}$ However, there are different aspects to consider for good survival of plastic cannulae, such as selecting the puncture sites through a rope ladder, area technique or buttonhole. ${ }^{12}$ The insertion of plastic cannulas into the AVF differs considerably from the procedure performed with metal needles. Plastic cannulas are inserted into the sites of the AVF via an introducer needle. The tip of the introducer first pierces the skin and then the vessel. Unlike drilling with the traditional metal needle, the cannulator has less control over the cannulation success, such as the possibility of seeing blood reflux, because the plastic cannulae has an anti-reflux valve, so there is no danger of blood spilling out of the cannulae during this process. ${ }^{2}$ An Australian nocturnal haemodialysis programme ${ }^{13}$ described how, according to Pierratos ${ }^{14}$ first work, initially nurses used plastic cannulas, but they found them more difficult to insert, and therefore they preferred to use blunt metal needles with the buttonhole technique.

The technique of inserting the plastic cannulae is different from that of traditional metal needles. The cannulator should hold the cannulae further back from the AVF, as the cannulae have a longer hub. Therefore, any movement of the cannulator is transferred more strongly to the tip of the introducer into the vessel. ${ }^{1}$ In addition, plastic cannulas with introducers do not have wings like fistula needles, being more difficult to hold. A common problem identified by cannulators is that they may not have advanced the introducer far enough in the vessel before advancing into the cannulae; therefore, the cannulae cannot enter the lumen of the vessel. ${ }^{1}$ At the same time, the cannulator is unable to manipulate the cannulae after the introducer is removed, as failed cannulations may occur. ${ }^{15}$

In a recent study by Marticorena et al., ${ }^{16}$ the comparison between plastic cannulae and the metal needle favoured plastic cannulae with the relative risk of requiring intervention or infiltration during haemodialysis higher for metal needles. The study focused mainly on the costs of the two interventions favouring the use of plastic cannulae. The frequency of trauma associated with infiltration during dialysis was 2.3 times higher for metal needles than plastic cannulas. Complications were usually correlated to sudden involuntary movements of the patient. Common and frequent complications such as infiltration or trauma can be prevented by using plastic cannulas. In Marticorena et al.'s ${ }^{16}$ study is reported that the size of the aneurysms, yet present at the moment of cannulation, decreased in two 
Table I. Descriptive statistics: proportions of use of needle and cannulas stratified for the continent, centre type and centre size (number of hospital beds) (median (first to third quartile)).

\begin{tabular}{lclr}
\hline & No of centres & Needle & Cannulas \\
\hline Continent & & & $0.05(0.00-0.52)$ \\
$\quad$ Africa and America & 6 & $0.95(0.48-1.00)^{\mathrm{a}, \mathrm{b}}$ & $0.00(0.00-0.00)$ \\
$\quad$ Asia & 28 & $1.00(0.73-1.00)^{\mathrm{a}}$ & $0.10(0.00-0.20)$ \\
$\quad$ Australia & 17 & $0.90(0.85-1.00)^{\mathrm{a}, \mathrm{b}}$ & $0.10(0.00-0.30)$ \\
$\quad$ Europe & 243 & $0.90(0.40-1.00)^{\mathrm{b}}$ & \\
Centre type & & & $0.00(0.00-0.20)$ \\
$\quad$ Company-owned & 30 & $1.00(0.80-1.00)^{\mathrm{a}, \mathrm{b}}$ & $0.00(0.00-0.20)$ \\
$\quad$ Privately-owned & 48 & $1.00(0.80-1.00)^{\mathrm{a}}$ & $0.10(0.00-0.38)$ \\
$\quad$ Public & 216 & $0.90(0.40-1.00)^{\mathrm{b}}$ & \\
Centre size & & & $0.10(0.00-0.36)$ \\
$\quad<50$ & 39 & $0.90(0.60-1.00)^{\mathrm{a}, \mathrm{b}, \mathrm{c}}$ & $0.00(0.00-0.20)$ \\
$50-100$ & 91 & $1.00(0.80-1.00)^{\mathrm{a}, \mathrm{b}}$ & $0.20(0.00-0.80)$ \\
$\quad$ 100-150 & 90 & $0.80(0.20-1.00)^{\mathrm{c}}$ & $0.00(0.00-0.30)$ \\
I50-200 & 25 & $1.00(0.70-1.00)^{\mathrm{a}, \mathrm{b}, \mathrm{c}}$ & $0.0(0.00-0.20)$ \\
$>200$ & 49 & $1.00(0.80-1.00)^{\mathrm{b}}$ & \\
\hline
\end{tabular}

a,b,cSignificant differences among groups (one-way ANOVA, Tukey post hoc multiple comparisons, $p<0.05$ ); the same letter, in uppercase, are related to groups not significantly different.

patients using plastic cannulae, without any additional intervention (i.e. angioplasty of a flow obstructing stenotic area), or change in flows or pressure.

The present study aimed to investigate the common use of plastic cannulae versus metal for cannulation in dialysis units and any related complications focusing on side effects of cannulation, that is, haematoma, infiltration.

\section{Materials and methods}

The study is a cross-sectional survey. A questionnaire was created to investigate plastic cannulae versus metal needles for cannulation among haemodialysis nurses. The online questionnaire was sent out to the nurse members of the European Dialysis Transplant Nurse Association/European Renal Care Association (EDTNA/ERCA). EDTNA/ERCA is a multidisciplinary European association of healthcare professionals caring for people with kidney disease, but it also counts many members from other countries worldwide (Japan, Israel, USA, Russia, Australia). The questionnaire was anonymous, and participation was voluntary. Respondents agreed to the processing of their data. The questionnaire was created after reviewing the literature and testing the questionnaire among eight members of the Executive Committee group of EDTNA/ERCA, with a wide experience in renal nursing ( $\min 12$ years-max 25 years)

\section{Statistical analysis}

Descriptive statistics were performed using median (first and third quartiles) or percentages where appropriate. Needles or cannulas use differences from the continent
(Africa, America, Asia, Australia, Europe), centre size, organisation (public, privately-owned, company-owned) were tested using one-way ANOVA with Tukey post hoc test. Africa and America categories were merged due to low sample size. Multiple analysis of variance for paired data was performed to highlight infiltration/haematoma proportion in different HD phases in the presence of various venous access conditions. All statistical elaborations were performed using Excel 365 and SPSS 22.0.

\section{Results}

A total of 294 responses were collected. About 217 respondents $(73.8 \%)$ were females, and 77 (26.2\%) were males. Five participants $(1.7 \%)$ were under 25 years old, $60(20.4 \%)$ were $25-34$ years old, $90(30.6 \%)$ were 35 44 years old, $93(31.6 \%)$ were $45-54$ years old and 45 $(15.6 \%)$ were $\geqslant 55$ years old. About 198 respondents $(67.3 \%)$ were nurses, $62(21.1 \% \%)$ were Head nurses and the other $34(11.6 \%)$ covered different positions (e.g. nurse practitioner, nurse education, nurse specialist, nurse clinician, nursing director). About 24 subjects (8.1\%) had less than 4 years of experience in haemodialysis, $58(19.7 \%)$ had between 4 and 10 years of experience and $212(72.1 \%)$ had more than 10 years of experience in nephrology. Data regarding the centres' type and size in which the respondents were working are reported in Table 1.

The use of metal needles was widespread in all continents; the lowest median value was found in Europe and Australia (90\%), while in other continents, it showed median values of at least $95 \%$. ANOVA test did not show a significant difference in usage. Consequently, the use of plastic cannulas is much lower, showing the highest median 
value (10\%) for Australia and Europe; it is also interesting to note that the highest third quartile is related to Europe; this means that at least $25 \%$ of the European centres of the sample use plastic cannulas in at least $30 \%$ of cases. ANOVA test with Tukey post hoc comparisons showed that cannulas use in Europe was significantly higher than in Asia, whereas Australia and Africa and America showed an intermediate use. The use of plastic cannulas depended on the type of centre $(p<0.05)$ and were mainly used in public centres $($ median $=10 \%$; third quartile $=38 \%$ ). The lowest usage was found in privately-owned companies ( median $=0 \%$; third quartile $=20 \%$ ). Finally, the highest use $(p<0.05)$ of cannulas was in centres of $100-150$ hospital beds, followed by centres with less than 50 beds. The results described above are reported in Table 1.

The mean proportions of the five more frequent complications regarding arteriovenous fistulas (AVF) (deep AVF, elbow located AVF, stenosed AVF, tortuous AVF, newly created/fragile AVF) using needle or cannulas were compared at four different phases of the haemodialysis process: cannulation, HD treatment, needle removal and haemostasis. For the deep, stenosed and tortuous AVF, there were no differences in the proportions of adverse events between the use of metal needles and plastic cannulas. For the elbow located AVF, a higher proportion of complications were reported when using the metal needle to cannulate $(p<0.001)$ during HD treatment $(p=0.001)$. In newly created/fragile AVF, metal needle use led to higher proportions of adverse events in all phases compared to the use of cannulas: cannulation $(p=0.03)$, HD treatment $(p<0.001)$, needle removal $(p=0.04)$, haemostasis $(p=0.02)$. All comparisons are reported in Figure 1.

The reasons why cannulas were not used are shown in Figure 2; almost a third of respondents reported they were too expensive, about a quarter did not find a clear benefit compared to usual practice; about $17 \%$ found difficulties in the procedure using cannulas, while the remaining affirmed the need for further steps or fixation and security problems.

\section{Discussion}

This study represents the first survey conducted on nurses at an international level regarding the use of plastic cannulae versus metal needles. The introduction of the plastic cannulae dates back to the 1950 s when infiltration, scarring and infection were identified concerning the use of metal needles for peripheral venous access and it was 'imperative that a more reliable, safer device to administer treatments was invented' ${ }^{2,9}$ However, the introduction of plastic cannulas was met with resistance among nursing staff. This resistance still appears strong from the results represented in the study conducted. Smith and Schoch ${ }^{2}$ observed that novice kidney nurses with a background of peripheral cannulation in general wards found it relatively easy to learn how to insert plastic cannulas. On the contrary, nephrology nurses who had punctured with metal needles for years found it very challenging to learn this new technique. This observation is in line with the sample of nurses responding to our questionnaire: $72 \%$ had more than 10 years of experience, but only a small proportion of nurses used plastic cannulas: $16.9 \%$ of respondents that had not used plastic cannulae had difficulties with this procedure. Despite adequate training, these difficulties could be related to modifying the cannulation technique from that with a needle. Another factor may be the 'Perpetual novice', phenomenon that happens when personal, environmental or contextual factors influence the nurse's experience and confidence in cannulation. Smith and Schoch ${ }^{9}$ noted that when some of the staff had difficulty using plastic cannulas, they went back to metal needles rather than persevere with the plastic option.

Another important difference emerges between continents. In Asia, there was no report of the use of plastic cannulae, although the literature reports interesting experiences conducted in Japan on its use for over 20 years on about 300,000 patients. ${ }^{8}$ The continents that appear most affected by their use today are Australia and Europe. Possible resistance to the use of the plastic device includes the difference in cost between the two devices. Although both are available with the same measures (in gauge), the cost difference between the two for a patient having three sessions of HD per week increases using a plastic device, between EUR 374.40 and EUR 436.80 per patient per year. ${ }^{1}$ This difference could push several hospitals, particularly those managed by private individuals, to use a less expensive device, with less training, another factor leading to increased costs. When plastic cannulas were introduced into an Australian renal department, staff needed three times more training (18 months instead of 6 months). ${ }^{2}$ Moving from metal needles to plastic cannulas requires substantial additional training and education. The technique is different, requires practice and time to enable successful cannulation. It is also important that the educator persists and motivates the staff to continue using cannulas after the initial training to become proficient with the new technique. ${ }^{2}$

Despite the small number of nurses with cannulae experience who responded to this study, there is a statistically significant difference in favour of using the plastic cannulae due to fewer reported complications in elbow located AVF and in frail or newly created AVF. In addition, the high costs of plastic cannulae may be offset by the reduced complications. In a randomised controlled pilot study, ${ }^{16}$ the patient group who received metal needles had statistically significant differences in complications such as mechanical trauma and infiltrations during HD. Shi et al. ${ }^{17}$ reports that extravasation phenomena and risk of haematoma or aborted dialysis session are lower in the plastic cannula group than in metal needle group. The cost of the 


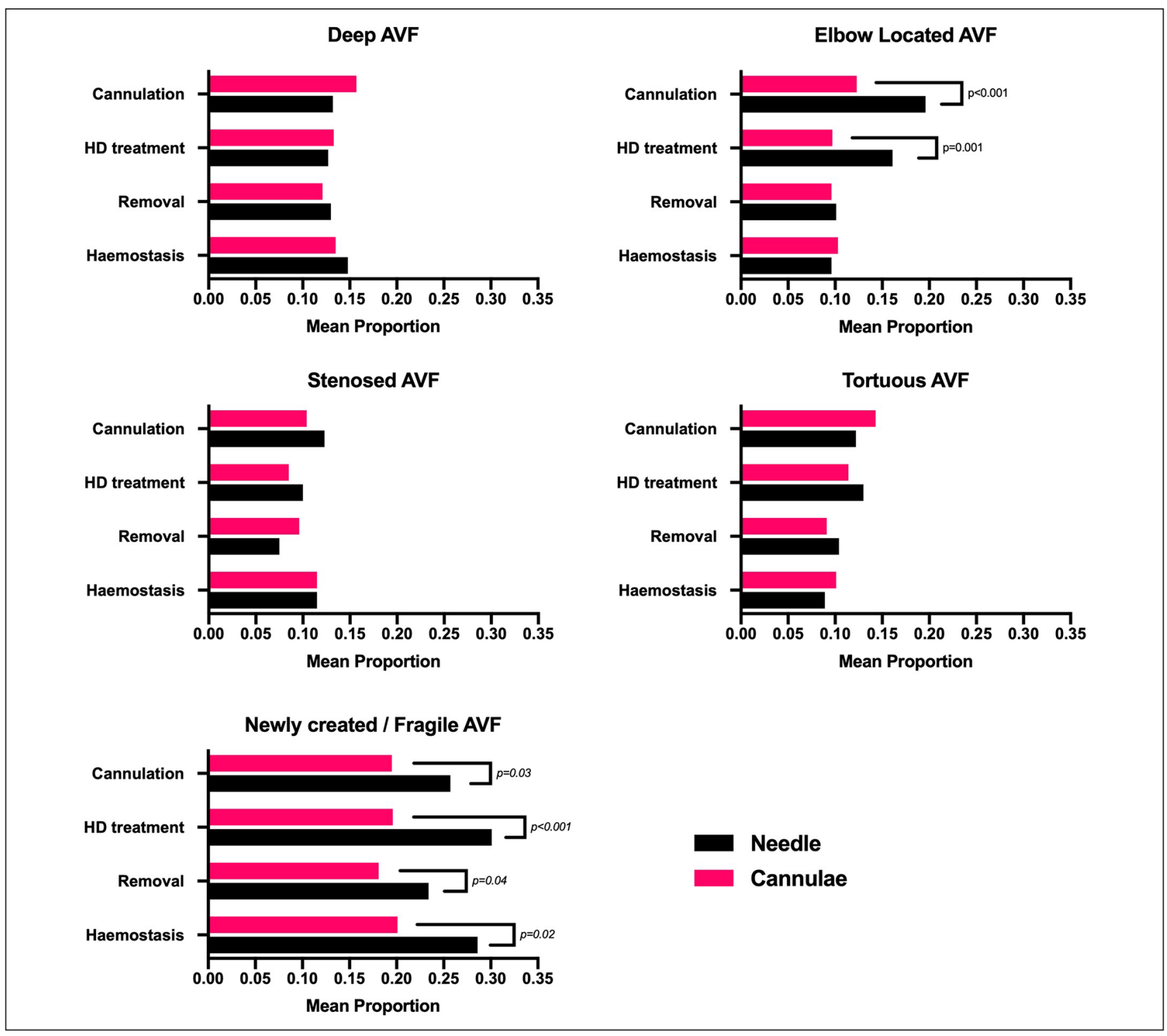

Figure I. Comparison of needle (black bars) and cannulas (red bars) in the more frequent AVF events (infiltration haematoma), during four phases of haemodialysis.

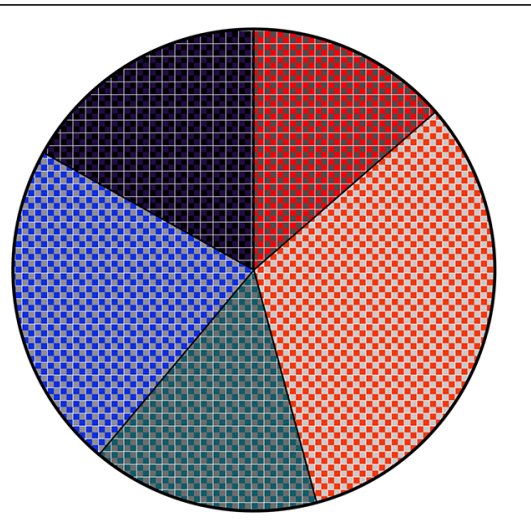

$16.9 \%$ Challenges in cannulation procedure

$22.0 \%$ No clear benefit vs current practice

15.3\% Cannulation: e.g. grip/needs of additional steps

$\square 32.2 \%$ Too expensive

$13.6 \%$ Fixation or security

Figure 2. Reasons for not using cannulae. 
interventions related to the treatment of complications led to a large increase in expenditure for the group who received metal needles. The metal needle group required a total of 22 additional procedures directly related to cannulation's consequences, while the plastic group had 11 procedures representing costs of $\$ 55,000$ and $\$ 27,500$, respectively, for patients. The total estimated cost/patientmonth was $\$ 6622$ for the metal group and $\$ 3787$ for the plastic cannulae group. ${ }^{10}$ Therefore, referring to the unit cost of cannulas is not useful for companies that want to achieve savings in the long run and a considerable favourable impact on the patient in terms of well-being.

The results obtained support what Du Toit ${ }^{8}$ reported on the use of plastic cannulae in regions that are 'difficult' to cannulate, such as the elbow area. Indeed, a statistically significant difference, based on the experience of the responding nurses, appears in favour of the plastic device. The other statistically significant difference that appears from the use of the plastic cannulae turns in favour of the newly created AVF: here too in the literature, the experiences of Letachowicz et al. ${ }^{18}$ and Choi et al. ${ }^{19}$ support what is shared by nurses about the minor complications deriving from the use of plastic cannulae on newly created AVF, and in particular in the upper arm AVF. ${ }^{19}$ The widespread use of plastic catheters can be useful particularly for elderly patients with multiple comorbidities and difficulties with coordination.

The studies report results in line with the conclusions of Nardinocchi et al..$^{20}$ who argues that: the introduction of this device in dialysis units must be accompanied by the use of operational protocols and adequate staff training ${ }^{19}$ to fill specific skills gaps, which should be identified through specific questionnaires. Change can be difficult but to research and analyse the past experiences of other professionals can help with the implementation of new devices in the world of haemodialysis. Ultimately this can be a step towards promoting personalised care, centred on the individual patient.

The study presents some limitations: the sample of respondents is a convenience sample with a clear European prevalence due to membership in the EDTNA/ERCA. The sample is not uniform in terms of length of service or experience in nephrology. The questionnaire did not specifically ask which area of the continent, or which state that historically have been more advanced in the use of the plastic cannulae, the respondents belonged to: therefore, it is not possible to say which states did not participate in the survey. No equal nurses used the two devices, with a predominant population using the metal needle. It is necessary to investigate complications with direct studies on the patient and have a representative number of both groups. The study, however, is the first international multicentre study, exploring the common use of plastic cannulae versus metal needles for cannulation in dialysis units, the implications focusing on side effects of cannulation and it includes nurses from various clinical settings. A statistically significant difference in favour of using the plastic cannulae emerged in the case of elbow located AVF and in frail or newly created AVF due to fewer complications. Further studies are needed to understand the differences in using the two devices within the same continent or between different states or units. It would also be interesting to analyse the reason for the greater use of plastic cannulae in public hospitals and if it is linked to continental factors (such as the greater presence of public versus private hospitals, health policies, etc.), rather than to the greater representation of the public compared to the private sector.

\section{Declaration of conflicting interests}

The author(s) declared no potential conflicts of interest with respect to the research, authorship, and/or publication of this article.

\section{Funding}

The author(s) disclosed receipt of the following financial support for the research, authorship, and/or publication of this article: Medtronic ${ }^{\circledR}$ supported the survey as part of the collaboration with EDTNA/ERCA.

\section{Ethical approval}

All procedures performed were following the ethical standards of the institutional research committee and with the 1964 Helsinki Declaration and its later amendments.

\section{Consent for publication}

All authors give their consent for publication.

\section{ORCID iDs}

Ilaria de Barbieri (iD https://orcid.org/0000-0002-7006-0733

Stefano Amatori (iD https://orcid.org/0000-0001-7497-755X

\section{References}

1. Parisotto MT, Pelliccia F, Bedenbender-Stoll E, et al. Haemodialysis plastic cannulae: a possible alternative to traditional metal needles? J Vasc Access 2016; 17: 373-379.

2. Smith V and Schoch M. Plastic cannula use in hemodialysis access. J Vasc Access 2016; 17: 405-410.

3. Donnelly SM and Marticorena RM. When is a new fistula mature? The emerging science of fistula cannulation. Semin Nephrol 2012; 32: 564-571.

4. Brescia MJ, Cimino JE, Appel K, et al. Chronic hemodialysis using venipuncture and a surgically created arteriovenous fistula. New Engl J Med 1966; 275: 1089-1092.

5. Twardowski $Z$ and Kubara H. Different sites versus constant sites of needle insertion into arteriovenous fistulas for treatment by repeated dialysis. Nephrol Dial Transplant 1979; 8: 978-980.

6. Di Nicolò $\mathrm{P}$, Cornacchiari $\mathrm{M}$, Mereghetti $\mathrm{M}$, et al. Buttonhole cannulation of the AV Fistula: a critical analysis of the technique. Semin Dial 2017; 30: 32-38. 
7. Grainer F. Plastic (non-metal) fistula cannula: from concept to practice. Renal Soc Aust J 2014; 10: 44-46.

8. Du Toit D. Haemodialysis needles: why do we use metal fistula needles? Renal Soc Aust J 2013; 9: 138-140.

9. Smith V and Schoch M. Do plastic cannulas have better outcomes than metal needles in haemodialysis? A retrospective review. Renal Soc Aust J 2017; 13: 78-82.

10. Nakai S, Iseki K, Itami N, et al. An overview of regular dialysis treatment in Japan (as of 31 December 2010). Ther Apher Dial 2012; 16: 483-521.

11. Centre for Healthcare Related Infection Surveillance and Prevention and Tuberculosis Control (CHRISP). Peripheral intravenous cannulation guideline V2, https://www.health. qld.gov.au/_data/assets/pdf_file/0025/444490/icare-pivcguideline.pdf $(2013$, accessed 9 October 2021).

12. Parisotto MT, Schoder VU, Miriunis C, et al. Cannulation technique influences arteriovenous fistula and graft survival. Kidney Int 2014; 86: 790-797.

13. Agar J. Frequently asked questions: "how are the needles secured and protected during NHHD?," http://www.nocturnaldialysis.org/faq pages.html\#20a (2012, accessed 6 December 2021).

14. Pierratos A. Nocturnal home haemodialysis: an update on a 5-year experience. Nephrol Dial Transplant 1999; 14: $2835-2840$.
15. Smith V. Transitioning to the new plastic cannula for dialysis cannulation of new arteriovenous fistulas: a training perspective, http://www.renalsociety.org/public/6/files/documents/ covidien/PosterPresentation-ANZSNRSAAug2014_Smith. $\operatorname{pdf}(2014$, accessed 9 October 2021).

16. Marticorena RM, Dacouris N and Donnelly SM. Randomized pilot study to compare metal needles versus plastic cannulae in the development of complications in hemodialysis access. J Vasc Access 2018; 19: 272-282.

17. Shi K, Jiang H and Wakabayashi M. Effect of early cannulation with plastic cannula on arteriovenous fistula patency in hemodialysis patients. Blood Purif 2020; 49: 79-84.

18. Letachowicz K, Kusztal M, Gołębiowski T, et al. Use of plastic needles for early arteriovenous fistula cannulation. Blood Purif 2015; 40: 155-159.

19. Choi YS, Lee HS, Joo N, et al. Efficacy and safety of plastic cannulae compared with metal needles in the initial use of an arteriovenous fistulae in incident hemodialysis patients: a randomized controlled study. Am J Nephrol 2021; 52(6): 479-486.

20. Nardinocchi MD, Manocchi K and Traini T. The appropriateness of cannulation devices in the management AVF. G Ital Nefrol 2020; 37(3): 10. 